latory equivalent for $\mathrm{CO}_{2}$ at anaerobic threshold $\left(\mathrm{EqCO}_{2} \mathrm{AT}\right)$, are increasingly being used as prognostic markers in heart failure and pulmonary arterial hypertension (PAH). Little is known about whether these measures can be applied to all forms of $\mathrm{PH}$, in particular chronic thromboembolic pulmonary hypertension (CTEPH), where thrombotic vascular occlusion may an impact on gas exchange through increased dead space fraction.

Methods and results 127 patients, 77 with CTEPH and 50 with PAH, underwent incremental CPX. Ventilatory dead space fraction $\left(V_{D} /\right.$ $\mathrm{V}_{\mathrm{T}}$ ) measured at peak exercise with arterial blood gas analysis was higher in CTEPH than PAH (52.9 vs 41.8, $\mathrm{p}<0.001$ ). The $\mathrm{V}_{\mathrm{E}} / \mathrm{VCO}_{2}$ slope was higher in CTEPH patients than in PAH patients (50.7 vs $44.4, \mathrm{p}=0.024)$ and was mirrored by similar changes in $\mathrm{EqCO}_{2} \mathrm{AT}$ (47.7 vs $42.0 \mathrm{p}=0.008$ ). Multivariate analysis demonstrated disease subtype to be a powerful independent predictor of $\mathrm{V}_{\mathrm{D}} / \mathrm{V}_{\mathrm{T}}$ $(\mathrm{p}<0.001), \mathrm{V}_{\mathrm{E}} / \mathrm{VCO}_{2}$ slope $(\mathrm{p}=0.003)$ and $\mathrm{EqCO}_{2} \mathrm{AT}(\mathrm{p}<0.001)$. These three measures could distinguish between WHO functional classes I/II and III/IV in PAH, but not CTEPH (Abstract S98 Figure 1). As a result of increased ventilatory inefficiency in CTEPH, breathing reserve was lower at peak exercise compared with $\mathrm{PAH}$ (29.0 vs $38.8, \mathrm{p}=0.003$ ) despite similar peak $\mathrm{VO}_{2}$ and heart rate reserves.

Conclusion Significant differences in gas exchange exist between CTEPH and PAH, possibly due to differences in $V_{D} / V_{T}$ as a result of vascular occlusion due to thromboembolic disease. These findings increase our understanding of the mechanisms of exercise limitation in subtypes of pulmonary hypertension, with increased dependence on gas exchange and ventilatory capacity in CTEPH. Furthermore these differences in gas exchange dissociate measures of ventilatory efficiency from disease severity in CTEPH. Caution should be applied in using common prognostic end-points from CPX in all forms of pulmonary hypertension.

\section{S99 MULTIPLE REGRESSION ANALYSES IN A COHORT OF HEREDITARY HAEMORRHAGIC TELANGIECTASIA PATIENTS SUGGEST A NOVEL ROLE FOR IRON IN THROMBOSIS}

doi:10.1136/thx.2010.150938.50

\footnotetext{
${ }^{1} \mathrm{~J}$ A Livesey, ${ }^{2} \mathrm{~J}$ E Jackson, ${ }^{1} \mathrm{C}$ L Shovlin. ${ }^{1} \mathrm{NHLI}$, Imperial College London, London, UK; ${ }^{2}$ Department of Imaging Imperial College Healthcare Trust, London, UK
}

Introduction/objectives Pulmonary emboli (PE) secondary to deep venous thromboses (DVT) are the immediate cause of $10 \%$ of hospital deaths. Elevated basal plasma coagulation factor (F) VIII levels, now known to be synthesised by the pulmonary endothelium, ${ }^{1}$ are the strongest predictor of recurrent PE/DVT, and also associated with elevated pulmonary artery pressure (PAP). The reasons for these associations are unknown. The study objectives were to determine whether novel patient-specific factors were associated with FVIII levels; PE/DVT; and/or PAP.

Methods Previous studies from our group have presented data on the 1999-2005 hereditary haemorrhagic telangiectasia (HHT) cohort of patients. In the current study, a replicate data series was generated from individuals seen between 2006 and 2010, and analysed independently to the first. Basal plasma FVIII measurements taken at least 4 months from any thrombotic event/intercurrent illness; and 32 patient-specific variables from 221 individuals, including cathetermeasured PAP and verified DVT/PE, were entered into an Excel chart. Following verification, data were analysed using STATA v11c (Statacorp LP) software. Univariate analyses identified potentially associated variables, which were analysed using stepwise multiple regression analyses, to generate best-fit models.

Results For FVIII, the most significant independent variables were identified as fibrinogen, age, and iron replacement therapy (IRT) (model: 4df; $\mathrm{p}<0.0001 ; \mathrm{r}^{2}$ 0.2014). Fibrinogen showed the most significant association with FVIII (OR 0.193, $\mathrm{p}<0.001$ ). FVIII showed further associations with age (OR 0.006, $p=0.028)$ and IRT (OR 0.162; $\mathrm{p}=0.075$ ). PE/DVT was associated with IRT (OR 1.416, $\mathrm{p}=0.028)$. Mean PAP was associated with age (OR 0.079, $\mathrm{p}=0.007$ ) and FVIII (OR 1.449, $\mathrm{p}=0.009$ ). Iron deficiency did not emerge as significant in any model. SIREs software predicted specific iron response elements (IREs) in FVIII mRNA splice-variants, including one in the $5^{\prime}$ UTR of splice-variant $2 .^{1}$

Conclusions This study suggests that basal plasma FVIII levels, and associated clinical endpoints of PE/DVT and PAP, may be influenced by iron replacement therapy, possibly by modulating expression of specific splice-variants of the F8 gene. This study therefore suggests that iron replacement may increase thrombotic risk, with significant potential clinical implications for millions of people who take iron tablets.

\section{REFERENCE}

1. Shovlin, et al. PLOS ONE 2010:5:e9154.

\section{S100 CHANGE IN DIASTOLIC PULMONARY ARTERY PRESSURE (3 MONTHS POST SURGERY COMPARED TO PRE-SURGERY VALUE) AS A LONG-TERM PROGNOSTIC PARAMETER IN PATIENTS TREATED WITH PULMONARY ENDARTERECTOMY FOR CHRONIC THROMBOEMBOLIC PULMONARY HYPERTENSION}

doi:10.1136/thx.2010.150946.1

G C Hagan, C Treacy, K Sheares, D P Jenkins, J PepkeZaba, A Kacprzak. Papworth Hospital, PapworthEverard, UK

Introduction The definitive treatment for chronic thromboembolic pulmonary hypertension is pulmonary endarterectomy (PEA). Some patients are left with residual pulmonary hypertension after the surgery. We analysed post-PEA haemodynamics to assess their impact on long-term survival.

Methods We analysed systolic(sPAP), diastolic(dPAP) and mean (mPAP) pulmonary artery pressures, pulmonary vascular resistance (PVR) and changes $(\Delta)$ in these parameters measured at right heart catheterisation from pre-PEA to 3 months post PEA in 251 patients who underwent PEA in our centre in years 2000-2009. We assessed their usefulness as survival predictors using receiver operating characteristic (ROC) curves for 56 patients who completed at least 6 years follow-up. Eight of them died during this period, 48 survived. Subsequently, on the basis of ROC, we identified a cut-off value for change in diastolic pulmonary artery pressure $(\triangle \mathrm{dPAP})$, the parameter which had the biggest area under the curve (AUC). We divided the whole study population according to that value and performed Kaplan-Meier survival analysis.

Results AUCs were as follows: $\operatorname{sPAP}(0.57), \Delta \mathrm{sPAP}(0.7), \operatorname{dPAP}(0.72)$, $\Delta$ dPAP(0.76), mPAP(0.66), $\Delta$ mPAP $(0.71), \operatorname{PVR}(0.65), \Delta \mathrm{PVR}(0.73)$ We chose $24.5 \mathrm{~mm} \mathrm{Hg}$ as a cut-off value for change in dPAP. It was characterised by $100 \%$ specificity and $41.9 \%$ sensitivity in predicting death within 6 years post-PEA. Kaplan-Meier survival analysis (with time zero taken at time of right heart catheter 3 months postPEA) for the whole group showed that patients with $\Delta$ dPAP $>24.5$ $\mathrm{mm} \mathrm{Hg}$ had significantly better survival rates in comparison to patients with $\Delta \mathrm{dPAP}<24.5 \mathrm{~mm} \mathrm{Hg}(\mathrm{p}=0.03)$ - Abstract S100 Figure 1. 13 of 59 patients with $\Delta$ dPAP $>24.5 \mathrm{~mm} \mathrm{Hg}$ still had pulmonary hypertension 3 months post-PEA. The overall 5 -year survival of the whole group was $80.8 \%$.

Conclusions 3 months post-PEA dPAP and $\triangle$ dPAP from pre-PEA values appeared to be the best survival predictor. The advantage of utilising dPAP is that it is a directly measured value and less susceptible to error then derived PVR. 


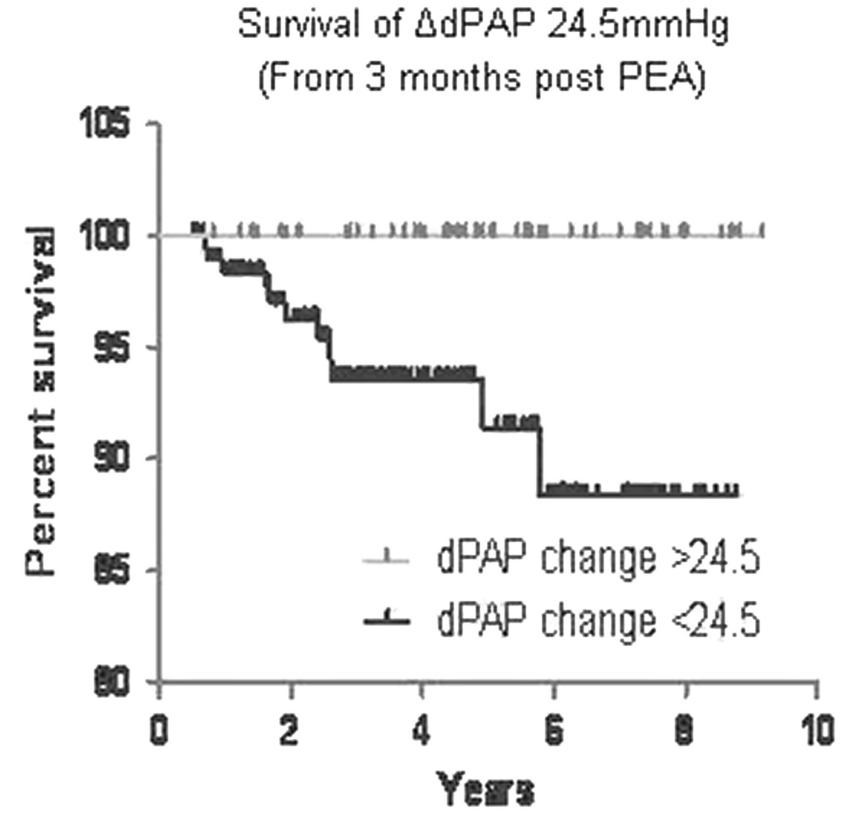

$\begin{array}{llllll}\text { IAPAP }>245 & 59 & 90 & 3 & 19 & 6 \\ \text { IAPAP }<245 & 192 & 130 & 63 & 26 & 7\end{array}$

Abstract S100 Figure 1 Survival of $\triangle \mathrm{dPAP} 24.5 \mathrm{~mm} \mathrm{Hg}$ (From 3 months post PEA).

\section{S101 PRAZIQUANTEL PREVENTS PROGRESSION AND REVERSES PULMONARY HYPERTENSION AND PULMONARY VASCULAR REMODELLING IN A MOUSE MODEL OF SCHISTOSOMIASIS}

doi:10.1136/thx.2010.150946.2

${ }^{1} \mathrm{~A}$ Crosby, ${ }^{1} \mathrm{~F} M$ Jones, ${ }^{2} \mathrm{E}$ Kakolos, ${ }^{1} \mathrm{M}$ Southwood, ${ }^{1} \mathrm{I}$ Purvis, ${ }^{2} \mathrm{G}$ Butrous, ${ }^{1} \mathrm{D}$ W Dunne, ${ }^{1} \mathrm{~N}$ Morrell. ${ }^{1}$ Cambridge University, Cambridge, UK; ${ }^{2}$ University of Kent, Canterbury, UK

Introduction Schistosomiasis is the most common world-wide cause of pulmonary hypertension. Praziquantel is the drug of choice and has been shown to reverse the liver pathology associated with Schistosomamansoni in mice. We sought to determine whether praziquantel could reverse established pulmonary vascular remodelling and pulmonary hypertension in a mouse model of Schistosomamansoni.

Methods Mice were infected percutaneously with a low dose of Schistosomamansoni. At 17 weeks post-infection mice were either sacrificed or received praziquantel by oral gavage or a vehicle control. At 17 or 25 weeks post-infection right ventricular systolic pressure (RVSP) and right ventricular (RV) hypertrophy, liver and lung egg counts were measured. Pulmonary vascular remodelling was assessed by morphometry, following immunohistochemistry. A cytokine array was performed and the degree of infectivity was measured by faecal egg counts.

Measurements and main results At 25 weeks post-infection there was a significant increase in RVSP and RV hypertrophy between infected and control mice, which was reversed by Praziquantel treatment. RVSP was elevated in mice at 25 weeks post-infection but had normalised with praziquantel treatment. There was a significant increase in the muscularisation of small pulmonary arteries following 25 weeks of schistosomal infection, which was prevented by with Praziquantel treatment. Liver, lung and faecal egg counts were elevated following 25 weeks of schistosomal infection and substantially reduced with praziquantel treatment.

Conclusions This study has shown that severe pulmonary vascular remodelling accompanied by an increase in RVSP and RV hypertrophy occurs 25 weeks post-infection in a mouse model of $\mathrm{S}$ mansoni infection. Importantly, this study has shown that progression of disease can be prevented by two oral doses of praziquantel. The mechanism thought to underlie the dramatic pulmonary vascular remodelling, is a local increase in inflammatory cytokines.

\section{Acute lung injury: what are the causes? S102 KGF ENHANCES PULMONARY PRODUCTION OF PRO- EPITHELIAL REPAIR FACTORS IN A HUMAN IN VIVO MODEL OF ACUTE LUNG INJURY}

doi:10.1136/thx.2010.150946.3

${ }^{1} \mathrm{M}$ Shyamsundar, ${ }^{1} \mathrm{C} \mathrm{M}$ O'Kane, ${ }^{2} \mathrm{C}$ Calfee, ${ }^{1} \mathrm{~S} \mathrm{~T}$ McKeown, ${ }^{1} \mathrm{C}$ Taggart, ${ }^{2} \mathrm{M}$ A Matthay, ${ }^{1} \mathrm{D}$ F McAuley. ${ }^{1}$ Centre for Infection and Immunity, Queen's University of Belfast, Belfast, Northern Ireland; '2University of California, San Francisco, San Francisco, USA

Introduction Keratinocyte Growth Factor (KGF) has been suggested as a possible intervention for acute lung injury. In vitro it enhances epithelial repair. In the human ex vivo perfused lung model KGF supplementation after LPS-induced injury was associated with improved alveolar fluid clearance indicating improved epithelial function. We hypothesised that KGF pre-treatment in the in vivo human LPS model of lung injury would reduce epithelial injury and increase production of mediators that induce epithelial repair.

Methods 36 subjects were randomised to either placebo or recombinant human KGF (Palifermin) $60 \mu \mathrm{g} / \mathrm{kg} /$ day for 3 days prior to inhalation of $50 \mu \mathrm{g} E$ Coli LPS. $6 \mathrm{~h}$ after LPS inhalation subjects underwent bronchoalveolar lavage (BAL). BAL concentrations of SPD, RAGE, MMP-9 and CRP were measured by ELISA. Total protein was measured by Bradford assay. Markers of LPS-induced pulmonary inflammation were measured using multiplex bead array (CXCL8, TNF $\alpha$, MCP-1, IL-6, MMP-7/-8) or ELISA (HMGB1 and Calgranulin C). Permeability was assessed by BAL IgG:total protein ratio.

Results KGF increased BAL SP-D (Abstract S102 Figure 1A) but not RAGE. KGF increased BAL MMP-9 by $77 \%(p=0.04)$. KGF increased BAL IL- 6 by $84 \%(p=0.03$ ) and CRP (Abstract $\mathrm{S} 102$ Figure $1 \mathrm{~B})$. KGF did not alter BAL total white cell count or neutrophilia, TNF $\alpha$, CXCL8, MCP-1, HMGB1 or Calgranulin C. KGF did not alter BAL total protein or permeability index. Increased frequency of altered taste or tongue sensation ( $p=0.01)$ and of facial erythema $(p=0.005)$ were observed in the KGF-treated group. There were no serious adverse events.
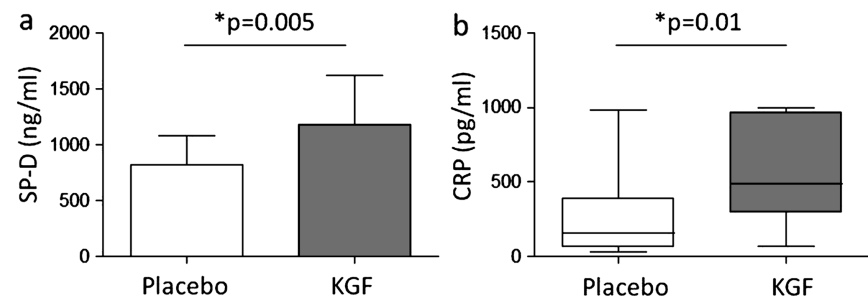

Abstract S102 Figure 1

Conclusion KGF specifically increased the production of several factors that are key for epithelial migration and wound healing including MMP-9 and IL-6, and increased the production of SP-D, a marker of type II alveolar epithelial cell proliferation. Furthermore it increases the production of BAL CRP which in the pulmonary compartment acts as an opsonin, aiding the clearance of apoptotic cells (or bacteria). However, KGF pre-treatment did not alter the inflammatory infiltrate or permeability change in response to inhaled LPS. Data suggest that KGF may promote a healing environment within the damaged alveoli and support further investigation of KGF as a treatment for lung injury. 